\title{
Hyperbolic characteristics on star-shaped hypersurfaces
}

\author{
by \\ Chun-gen $\mathrm{LIU}^{1}$ and Yiming LONG ${ }^{2,3}$ \\ Nankai Institute of Mathematics, Nankai University, \\ Tianjin 300071, People's Republic of China
}

\begin{abstract}
In this paper, we study the stability of closed characteristics on a starshaped compact smooth hypersurface $\Sigma$ in $\mathbb{R}^{2 n}$. We show that the Maslov-type mean index of such a closed characteristic is independent of the choice of the Hamiltonian functions, and prove that on $\Sigma$ either there are infinitely many closed characteristics, or there exists at least one nonhyperbolic closed characteristic, provided every closed characteristic possesses its Maslov-type mean index greater than 2 when $n$ is odd, and greater than 1 when $n$ is even.

(C) 1999 L'Association Publications de l'Institut Henri Poincaré. Published by Elsevier B.V. All rights reserved
\end{abstract}

Key words: Hamiltonian systems, Maslov-type mean index, iterations, hyperbolic closed characteristics, star-shaped energy hypersurface

RÉSUMÉ. - Soit $\Sigma$ une hypersurface étoilée compacte, $C^{2}$, dans $\mathbb{R}^{2 n}$, qui est obtenue par la méthode de variation directe. Dans cet article, nous étudions la stabilité des caractéristiques fermées sur $\Sigma$. Nous démontrons que l'indice moyen de type de Maslov d'une telle caractéristique fermée ne dépend pas du choix des fonctions Hamiltoniennes. Si on suppose qu'il n'existe qu'un nombre fini de caractéristiques fermées, alors il existe au moins une caractéristique fermée nonhyperbolique sur $\Sigma$ si pour toute (C) 1999 L'Association Publications de l'Institut Henri Poincaré. Published by Elsevier B.V. All rights reserved

1991 AMS Math. Subj. Classification: 58F05, 58E05, 34C25, 58F10.

1. Partially supported by the Qiu Shi Sci. and Tech. Foundation.

2. Partially supported by NNSF and MCSEC of China and the Qiu Shi Sci. and Tech. Foundation.

3. Associate member of ICTP.

Annales de l'Institut Henri Poincaré - Analyse non linéaire - 0294-1449 
caractéristique fermée sur $\Sigma$, son indice moyen de type de Maslov est supérieur à 2, et si $n$ est impair (resp. 1, et si $n$ est pair). (C) Elsevier, Paris

\section{INTRODUCTION AND MAIN RESULTS}

In this paper, we consider the Maslov-type index theory for star-shaped Hamiltonian systems and generalize certain results of fixed energy problems on convex hypersurfaces of [10] and [21] to the fixed energy problems on star-shaped hypersurfaces in $\mathbb{R}^{2 n}$. We study the stability of closed characteristics on given compact $C^{2}$ hypersurfaces in $\mathbb{R}^{2 n}$ with $n \geq 2$ bounding a star-shaped set with nonempty interior.

A $C^{2}$ compact hypersurface $\Sigma$ in $\mathbb{R}^{2 n}$ is star-shaped, if it bounds an open set $\Gamma(\Sigma)$, and there exists a point $x_{0}(\Sigma) \in \Gamma(\Sigma)$ such that the tangent plane of $\Sigma$ at any point $x \in \Sigma$ does not passing though $x_{0}(\Sigma)$. We denote by $\mathcal{S}\left(\mathbb{R}^{2 n}\right)$ the set of all such hypersurfaces in $\mathbb{R}^{2 n}$ and by $\mathcal{S}_{0}\left(\mathbb{R}^{2 n}\right)$ the subset of all such $\Sigma$ with $x_{0}(\Sigma)=0$. For $\Sigma \in \mathcal{S}\left(\mathbb{R}^{2 n}\right)$, without loss of generality we suppose $x_{0}(\Sigma)=0$. Let $j_{\Sigma}: \mathbb{R}^{2 n} \rightarrow[0,+\infty)$ be the gauge function of $\Gamma(\Sigma)$ defined by

$$
j_{\Sigma}(0)=0, \text { and } j_{\Sigma}(x)=\inf \left\{\lambda>0 \mid \frac{x}{\lambda} \in \Gamma(\Sigma)\right\} \text { for } x \in \mathbb{R}^{2 n} \backslash\{0\} .
$$

For $x \in \Sigma$ let $N_{\Sigma}(x)$ be the unit outward normal vector of $\Sigma$ at $x$. We consider the given energy problem of finding $\tau>0$ and an absolutely continuous curve $x:[0, \tau] \rightarrow \mathbb{R}^{2 n}$ such that

$$
\left\{\begin{array}{l}
\dot{x}(t)=J N_{\Sigma}(x(t)), \quad x(t) \in \Sigma, \quad \forall t \in \mathbb{R} \\
x(\tau)=x(0)
\end{array}\right.
$$

where $J=\left(\begin{array}{cc}0 & -I_{n} \\ I_{n} & 0\end{array}\right)$ is the standard symplectic matrix on $\mathbb{R}^{2 n}$ with $I_{n}$ being the identity matrix on $\mathbb{R}^{n}$. The symplectic group is defined by $\mathrm{Sp}(2 n)=\left\{M \in \mathcal{L}\left(\mathbb{R}^{2 n}\right) \mid M^{T} J M=J\right\}$, where we denote by $M^{T}$ the transpose of $M$ and $\mathcal{L}\left(\mathbb{R}^{2 n}\right)$ the set of all $2 n \times 2 n$ real matrices. A nonconstant solution $(x, \tau)$ of (1.2) with $\tau>0$ being the minimal period of $x$ is called a closed characteristic on $\Sigma$. Denote by $\mathcal{J}(\Sigma)$ the set of all closed characteristics on $\Sigma$. The existence of at least one closed characteristic on any $\Sigma \in \mathcal{S}\left(\mathbf{R}^{2 n}\right)$ was first established by $\mathrm{P}$. Rabinowitz in his pioneering work [24] in 1978 . 
For a given $\Sigma \in \mathcal{S}\left(\mathbb{R}^{2 n}\right)$, we define a Hamiltonian function $H_{4}: \mathbb{R}^{2 n} \rightarrow$ $[0,+\infty)$ by

$$
H_{\alpha}(x)=j_{\Sigma}(x)^{\alpha}, \quad \forall x \in \mathbb{R}^{2 n}, \text { and } \alpha>1 .
$$

Then $H_{4} \in C^{2}\left(\mathbb{R}^{2 n}, \mathbb{R}\right)$ and $\Sigma=H_{4}^{-1}(1)$. It is well known that the problem (1.2) is equivalent to the following problem

$$
\left\{\begin{array}{l}
\dot{x}(t)=J H_{4}^{\prime}(x(t)), \quad H_{4}(x(t))=1, \quad \forall t \in \mathbb{R}, \\
x(\tau)=x(0) .
\end{array}\right.
$$

Denote by $\mathcal{J}_{4}(\Sigma)$ the set of all solutions $(x, \tau)$ of the problem (1.4) with $\tau$ being the minimal period of $x$. Note that $\mathcal{J}(\Sigma)$ and $\mathcal{J}_{4}(\Sigma)$ are 1-1 correspondent to each other. For $(x, \tau) \in \mathcal{J}_{4}(\Sigma)$, the linearized system of $(1.4)$ at $(x, \tau)$ is given by

$$
\left\{\begin{array}{l}
\dot{y}(t)=J H_{4}^{\prime \prime}(x(t)) y(t), \quad \forall t \in \mathbb{R} \\
y(\tau)=y(0)
\end{array}\right.
$$

The fundamental solution $\gamma_{x}$ of (1.5) is a path in $\operatorname{Sp}(2 n)$ starting from $I_{2 n}$. There is a Maslov-type index theory on $\gamma_{x}$ which was defined as an integer pair $\left(i_{\tau}(x), \nu_{\tau}(x)\right)$ (cf: [5], [23], [17], [19], and [22]).

In order to get the periodic solutions of problem (1.4), we consider the fixed period problem of the following star-shaped Hamiltonian system

$$
\left\{\begin{array}{l}
\dot{x}(t)=J H_{4}^{\prime}(x(t)) \\
x(1)=x(0)
\end{array}\right.
$$

This problem is equivalent to finding the critical points of the following action functional on the Hilbert space $E=W^{1 / 2,2}\left(S^{1}, \mathbb{R}^{2 n}\right)$

$$
f(x)=\frac{1}{2} \int_{0}^{1}(-J \dot{x}, x) d t-\int_{0}^{1} H_{4}(x(t)) d t, \quad \forall x \in E .
$$

The fundamental solution matrix $\gamma_{x}$ of (1.5) is a path in $\operatorname{Sp}(2 n)$ starting from $I_{2 n}$. The Floquet multipliers of $(x, \tau)$ are defined to be the eigenvalues of $\gamma_{x}(\tau)$. By Lemma 3.3 below, the Floquet multipliers with their multiplicity and Krein signs of $(x, \tau) \in \mathcal{J}_{4}(\Sigma)$ do not depend on the particular choice of the Hamiltonian function in (1.6). Thus the following definition makes sense.

DeFINITION 1.1. - A closed characteristic $(x, \tau) \in \mathcal{J}_{4}(\Sigma)$ is hyperbolic if as a solution of (1.4), 1 is a double Floquet multiplier of $\gamma_{x}(\tau)$ and all other Vol. 16, n $n^{\circ}$ 6-1999. 
Floquet multipliers of $\gamma_{x}(\tau)$ are not on the unit circle $\mathbf{U}$ in the complex plane $\mathbb{C}$. It is elliptic, if all the Floquet multipliers of $\gamma_{x}(\tau)$ are on $\mathbf{U}$.

For any $(x, \tau) \in \mathcal{J}_{4}(\Sigma)$ and $m \in \mathbb{N}=\{1,2, \cdots\}$, the $m$-th iteration $x^{m}$ of $x$ is defined by

$$
x^{m}(t)=x(t-j \tau) \text { for } j \tau<t \leq(j+1) \tau, \quad 0 \leq j \leq m-1
$$

This is simply $x$ itself viewed as an $m \tau$-periodic function. The Maslovtype index theory assigns to the iteration sequence $\left\{x^{m}\right\}$ of each solution $(x, \tau) \in \mathcal{J}_{4}(\Sigma)$ a sequence of integers $\left\{\left(i_{m \tau}\left(x^{m}\right), \nu_{m \tau}\left(x^{m}\right)\right)\right\}_{m \in \mathbb{N}}$ through the associatedd symplectic path $\gamma_{x}$ of $x$. The Maslov-type mean index of $x$ per period $\tau$,

$$
\hat{i}_{\tau}(x):=\lim _{m \rightarrow \infty} \frac{i_{m \tau}\left(x^{m}\right)}{m} .
$$

was first defined by the second author of this paper in [20]. In the section 3 , we prove that the Maslov-type mean index for closed characteristics on starshaped hypersurfaces is a geometric concept, it is independent of the choice of the Hamiltonian functions. ( $\mathrm{sec}$ Theorem 3.1 below).

Let $x$ be a nonconstant critical point of $f$ in $E, h=H_{4}(x)$, and $\frac{1}{m}$ be the minimal period of $x$ for some $m \in \mathbb{N}$. Define

$$
x_{\Sigma}(t)=h^{-\frac{1}{4}} x\left(h^{-\frac{1}{2}} t\right) \text { and } \tau=\frac{1}{m} h^{\frac{1}{2}} .
$$

Then there hold $x_{\Sigma}(t) \in \Sigma$ for all $t \in \mathbb{R}$ and thus $\left(x_{\Sigma}, \tau\right) \in \mathcal{J}_{4}(\Sigma)$. Note that the period 1 of $x$ corresponds to the period $m \tau$ of the solution $\left(x_{\Sigma}^{m}, m \tau\right)$ of (1.4) with minimal period $\tau$.

On the other hand, every solution $(x, \tau) \in \mathcal{J}_{4}(\Sigma)$ gives rise to a sequence $\left\{x_{m}\right\}_{m \in \mathbb{N}}$ of solutions of the problem (1.6), which is also a sequence of critical points of $f$ in $E$ :

$$
x_{m}=(m \tau)^{\frac{1}{2}} x(m \tau t), \quad \forall m \in \mathbb{N} .
$$

THEOREM 1.2. - On every $\Sigma \in \mathcal{S}\left(\mathbb{R}^{2 n}\right)$, either there exist infinitely many closed characteristics, or there exists at least one non-hyperbolic closed characteristic, provided every closed characteristic on $\Sigma$ possesses its Maslov-type mean index greater than 2 when $n$ is odd, and greater than 1 when $n$ is even.

This result is related to the works on the stability problem of I. Ekeland in [11] and of Y. Long in [21], as well as the problem 3 proposed at the end 
of Ekeland's celebrated book [10] in 1990. Up to the authors' knowledge, it seems that except our above theorem on the star-shaped hypersurfaces so far all the other stability results for closed characteristics obtained by variational methods on given energy hypersurfaces are only proved for convex (or similarly for concave) cases. We refer also the readers to works of I. Ekeland, G. Dell'Antonio, and B. D'Onofrio in [6], [7], and [12], and the references therein.

\section{THE MASLOV-TYPE INDEX AND THE GALERKIN APPROXIMATION}

Let $\Sigma$ be a compact $C^{2}$ hypersurface in $\mathbb{R}^{2 n}$ strictly star-shaped with respect to the origin. We shall consider the closed characteristics of $\Sigma$ which is the periodic solution of

$$
\dot{x}=J N_{\Sigma}(x),
$$

where $N_{\Sigma}(x)$ is the outward normal, normalized by the condition $\left(N_{\Sigma}(x), x\right)=1$, here $(\cdot, \cdot)$ denotes the inner product in $\mathbb{R}^{2 n}$.

If $H(x) \in C^{2}\left(\mathbb{R}^{2 n}, \mathbb{R}\right)$ is a function such that $\Sigma=H^{-1}(1)$ and 1 is a regular value of $H$, it is well known that the periodic solutions of (2.0) coincide with those periodic solutions of

$$
\dot{x}=J H^{\prime}(x), \quad x(t) \in \Sigma, \quad \forall t \in \mathbb{R} .
$$

We denote by $\Gamma(\Sigma)$ the open set bounded by $\Sigma$. Let $j_{\Sigma}: \mathbb{R}^{2 n} \rightarrow[0,+\infty)$ be the gauge function of $\Gamma(\Sigma)$ defined by

$$
j_{\Sigma}(0)=0 \text { and } j_{\Sigma}(x)=\inf \left\{\lambda \mid \frac{x}{\lambda} \in \Gamma(\Sigma)\right\} \text { for } x+0 .
$$

In this paper we choose the Hamiltonian function to be

$$
H(x)=H_{4}(x)=j_{\Sigma}(x)^{4}, \quad \forall x \in \mathbb{R}^{2 n} .
$$

It is well known that $H_{4} \in C^{2}\left(\mathbb{R}^{2 n}, \mathbb{R}\right)$ and $\Sigma=H_{4}^{-1}(1)$. In the following we consider the fixed energy problem

$$
\left\{\begin{array}{l}
\dot{x}(t)=J H_{4}^{\prime}(x(t)), \quad H_{4}(x(t))=1, \quad \forall t \in \mathbb{R}, \\
x(\tau)=x(0)
\end{array}\right.
$$

for $(x, \tau)$ with $\tau>0$. As usual, we set $E=W^{1 / 2,2}\left(S^{1}, \mathbb{R}^{2 n}\right)$, where $S^{1}=\mathbb{R} / \mathbb{Z}$. This is a Hilbert space whose norm and inner product are Vol. 16, $n^{\circ}$ 6-1999. 
denoted by $\|\cdot\|$ and $\langle\cdot, \cdot\rangle$ respectively. The space $E$ consists of all $z \in L^{2}\left(S^{1}, \mathbb{R}^{2 n}\right)$ whose Fourier series

$$
z(t)=a_{0}+\sum_{j=1}^{\infty}\left(a_{j} \cos (2 j \pi t)+b_{j} \sin (2 j \pi t)\right)
$$

satisfies

$$
\|z\|^{2}=\left|a_{0}\right|^{2}+\frac{1}{2} \sum_{j=1}^{\infty} j\left(\left|a_{j}\right|^{2}+\left|b_{j}\right|^{2}\right)<\infty,
$$

where $a_{j}, \quad b_{j} \in \mathbb{R}^{2 n}$. Let $L_{c}(E)$ and $L_{s}(E)$ denote the set of linearly compact operators and the set of bounded self-adjoint operators on $E$, respectively. For $B(t) \in C\left(S^{1}, L_{s}\left(\mathbb{R}^{2 n}\right)\right)$, we define two operators $A, \quad B \in L_{s}(E)$ by extending the bilinear forms

$$
\begin{aligned}
& \langle A x, y\rangle=\int_{0}^{1}(-J \dot{x}, y) d t \\
& \langle B x, y\rangle=\int_{0}^{1}(B(t) x, y) d t
\end{aligned}
$$

to $E$. Clearly, $\operatorname{ker} A=\mathbb{R}^{2 n} . A$ is a Fredholm operator with ind $A=0$, and $B \in L_{c}(E)$. Using the Floquet theory we have

$$
\nu_{1} \equiv \operatorname{dim} \operatorname{ker}\left(R(1)-I_{2 n}\right)=\operatorname{dim} \operatorname{ker}(A-B),
$$

where $R(t)$ is the fundamental solution of the linear Hamiltonian system

$$
\dot{y}=J B(t) y .
$$

$R(t)$ is a symplectic matrix for every time $t$ with $R(0)=I_{2 n}$. It is equipped with a pair of integers:

$$
\left(i_{1}, \nu_{1}\right) \in \mathbb{Z} \times\{0,1, \cdots, 2 n\},
$$

the Maslov-type index of $B(t)$ (cf. [5], [23], [17] and [22])

Let $\Gamma=\left\{P_{m}, m=0,1, \cdots\right\}$ be an usual Galerkin approximation frame with respect to $A$, i.e., $\Gamma$ is a sequence of orthogonal projections satisfying the following conditions:

(i) $P_{0} E=\operatorname{ker} A, E_{m}=P_{m} E$ is finite dimensional for $m \geq 1$.

(ii) $P_{m} P_{m+1}=P_{m+1} P_{m}=P_{m}, \forall m$.

(iii) $P_{m} x \rightarrow x$, as $m \rightarrow+\infty \forall x \in E$.

(iV) $P_{m} A=A P_{m}, \forall m$. 
We denote by $M_{d}^{+}(D), M_{d}^{-}(D)$ and $M_{d}^{0}(D)$ the eigenspaces of a self-adjoint operator $D$ corresponding to the eigenvalue $\lambda$ belonging to $[d,+\infty),(-\infty,-d]$ and $[-d, d]$, respectively for $d>0$. We also denote by $M^{+}(D), M^{-}(D)$ and $M^{0}(D)$ the positive, negative and null spaces of a self-adjoint operator $D$, respectively. For any $L \in L_{s}(E)$ we denote by $L^{\#}=\left(\left.L\right|_{\operatorname{Im} L}\right)^{-1}$ and $L_{m}=\left.\left(P_{m} L P_{m}\right)\right|_{P_{m} E}: P_{m} E \rightarrow P_{m} E$. When 0 is not an essential spectrum point of $L, L^{\#}$ is a bounded operator. We shall need the following result of T. Wang and G. Fei [28] (cf. also [13]).

LEMMA 2.1 (Theorem 2.1 of [28]). - For any $B(t) \in C\left(S^{1}, L_{s}\left(\mathbb{R}^{2 n}\right)\right)$ with the Maslov-type index $\left(i_{1}, \nu_{1}\right)$ and any constant $0<d<\frac{1}{4}\left\|(A-B)^{\#}\right\|^{-1}$, for large number $m$ we have

$$
\begin{gathered}
\operatorname{dim} M_{d}^{+}\left(P_{m}(A-B) P_{m}\right)=\frac{1}{2} \operatorname{dim}\left(P_{m} E\right)-i_{1}-\nu_{1}, \\
\operatorname{dim} M_{d}^{-}\left(P_{m}(A-B) P_{m}\right)=\frac{1}{2} \operatorname{dim}\left(P_{m} E\right)+i_{1}, \\
\operatorname{dim} M_{d}^{0}\left(P_{m}(A-B) P_{m}\right)=\nu_{1},
\end{gathered}
$$

where $B$ is the operator defined by (2.2) corresponding to $B(t)$.

\section{THE INVARIANCE OF THE MASLOV-TYPE MEAN INDEX AND THE RELATION WITH THE EKELAND MEAN INDEX}

Given a function $H \in C^{2}\left(\mathbb{R}^{2 n}, \mathbb{R}\right)$, we consider the Hamiltonian system

$$
\dot{x}=J H^{\prime}(x) \text {. }
$$

It is well known that every solution of (3.1) lie on some energy surface $\Sigma=\left\{x \in \mathbb{R}^{2 n} \mid H(x)=b\right\}$ for some $b \in \mathbb{R}$. Suppose $x \in C^{1}\left(\mathbb{R}, \mathbb{R}^{2 n}\right)$ is a $\tau$-periodic solution on the energy surface $\Sigma$. Replacing $H(x)$ by $H(x) / b$, without loss of generality, we suppose $b=1$. The linearized system of (3.1) at $x$ is defined by

$$
\dot{y}=J H^{\prime \prime}(x(t)) y \text {. }
$$

In this section, we denote by $\mathcal{E}\left(\mathbb{R}^{2 n}\right)$ the set of all energy surfaces $\Sigma$ which is a compact $C^{2}$ hypersurface in $\mathbb{R}^{2 n}$, bounding a domain with origin in its interior, and there exists a function $H$ satisfying the following conditions

(H1) $H \in C^{2}\left(\mathbb{R}^{2 n}, \mathbb{R}\right)$,

(H2) $\Sigma=\{x \mid H(x)=1\}$,

(H3) $\forall x \in \Sigma, H^{\prime}(x) \neq 0$,

(H4) $\forall x \in \Sigma, H^{\prime}(x)$ coincides with the outward normal direction of $\Sigma$. 
By the Lemmas 2 and 3 of [29], a compact hypersurface of contact type has a naturally detined "inside" and "outside". If a vector field $\eta$ on $\left(\mathbb{R}^{2 n}, \omega\right)$ is a symplectic dilation(i.e. $\left.\mathcal{L}_{\eta} \omega=\omega\right)$, and $H$ is a function having $\Sigma$ as a regular level surface, then $\langle d H, \eta\rangle=-\omega\left(\eta, X_{H}\right) \neq 0$. So if $\eta$ which is transverse to $\Sigma$ is outward and $\langle d H, \eta\rangle>0$, then $H^{\prime}(x)$ satisfies the condition (H4). Therefore, the case with conditions ( $\mathrm{H} 1)-(\mathrm{H} 4)$ include the contact type hypersurfaces. In [26] the existence of closed orbit on contact type hypersurface was proved. For $\Sigma \in \mathcal{E}\left(\mathbb{R}^{2 n}\right)$ we denote by $\mathcal{H}(\Sigma)$ the set of all the functions which satisfying the conditions (H1)-(H4), and denote by $\mathcal{J}(\Sigma, H)$ the set of all closed characteristics of $(3.1)$ on $\Sigma$. In the appendix of this paper we briefly review the $\omega$-index theory for symplectic paths starting from identity $I_{2 n}$. The main result of this section is the following invariant theorem about the Maslov-type mean index.

THEOREM 3.1. - For any $\Sigma \in \mathcal{E}\left(\mathbb{R}^{2 n}\right)$ and $H, G \in \mathcal{H}(\Sigma)$. If $(x, \tau) \in \mathcal{J}(\Sigma, H)$, then there exists a $C^{1}$-increasing diffeomorphism $\sigma:[0, \tau] \rightarrow[0, \mu]$ such that $\left(z_{\sigma}, \mu\right) \in \mathcal{J}(\Sigma, G)$ with $z_{\sigma}(t)=x\left(\sigma^{-1}(t)\right)$. We denote by $\hat{i}(\tau, x, H)$ and $\hat{i}\left(\mu, z_{\sigma}, G\right)$ the Maslov-type mean indices per period of the periodic solutions $(\tau, x)$ and $\left(\mu, z_{\sigma}\right)$ respectively. Then there holds

$$
\hat{i}(\tau, x, H)=\hat{i}\left(\mu, z_{\sigma}, G\right) .
$$

In order to prove Theorem 3.1, we need the following Lemmas.

Lemma 3.2. - Let $H, G \in \mathcal{H}(\Sigma)$ and $(x, \tau) \in \mathcal{J}(\Sigma, H)$. There is an increasing $C^{1}$-diffeomorphism $\sigma$ from $[0, \tau]$ onto an interval $[0, \mu]$ such that $\left(z_{\sigma}, \mu\right) \in \mathcal{J}(\Sigma, G)$ with $z_{\sigma}:=x\left(\sigma^{-1}(s)\right)$.

Proof. - We refer the readers to [24] and [10] for the details of the proof.

LEMMA 3.3. - Let $H$ and $G$ be the functions in Lemma 3.2. $(\tau, x)$ and $\left(\mu, z_{\sigma}\right)$ be defined in Lemma 3.2. Then $(\tau, x)$ and $\left(\mu, z_{\sigma}\right)$ have the same Floquet multipliers with the same multiplicity and the same Krein sign.

Proof. - The proof of this Lemma is the same as the proof of Proposition I.6.13 of [10] since the convex condition is not actually needed in that proof.

Consider the functions $H$ and $G \in \mathcal{H}(\Sigma)$. We have two linearized systems

$$
\dot{y}=J H^{\prime \prime}(x(t)) y
$$

and

$$
\dot{y}=J G^{\prime \prime}\left(z_{\sigma}(s)\right) y
$$


Let $R_{H}(t)$ and $R_{G}(s)$ be the fundamental solutions of (3.3) and (3.4) starting from the identity respectively. From Lemma $3.3, R_{H}(\tau)$ and $R_{G}(\mu)$ have the same Floquet multipliers with the same multiplicity and the same Krein sign. Using notations in the section 5, we denote the $\omega$-index $i_{\tau, \omega}\left(R_{H}\right)$ of the symplectic path $R_{H}$ in $\operatorname{Sp}(2 n)$ by $i_{\tau, \omega}(H)=i_{\tau, \omega}\left(R_{H}\right)$ as well as $i_{\mu, \omega}(G)=i_{\mu, \omega}\left(R_{G}\right)$ for $\omega \in \mathbf{U}=\{z \in \mathbb{C}|| z \mid=1\}$.

LEMMA 3.4. $-\mathcal{H}(\Sigma)$ is a nonempty convex set. i.e., if $H, G \in \mathcal{H}(\Sigma)$, then $(1-\lambda) H+\lambda G \in \mathcal{H}(\Sigma), \forall \lambda \in[0,1]$.

Proof. - By direct verification.

Note that the number of discontinuous points of $i_{\tau, \omega}(H)$ as a function of $\omega \in \mathbf{U}$ is bounded by $2 n$, and the integral formula of the mean index (cf. (5.9)), to prove Theorem 3.1 we start from the following result.

Proposition 3.5. - If $\omega \in \mathrm{U}$ and $\omega \notin \sigma\left(R_{H}(\tau)\right)=\sigma\left(R_{G}(\mu)\right)$, there holds

$$
i_{\tau, \omega}\left(\left.R_{H}\right|_{[0, \tau]}\right)=i_{\mu, \omega}\left(\left.R_{G}\right|_{[0, \mu]}\right)
$$

In order to prove this proposition, as in [10], we consider $i_{t, \omega}(\gamma)$ as a function of $t>0$. From the definition of $\omega$-index, we have the following result.

LEMmA 3.6. - If $\operatorname{det}(\gamma(t)-\omega I) \neq 0, \forall t \in\left[t_{0}, t_{1}\right]$, then

$$
i_{t_{0}, \omega}(\gamma)=i_{t_{1}, \omega}(\gamma)
$$

Proof. - Let $\sigma(t)=t_{1} t / t_{0}$, and $\phi(t)=\gamma \circ \sigma(t)$. Then $i_{t_{0}, \omega}(\gamma)=i_{t_{1}, \omega}(\phi)$ by the Theorem 5.4 of the $\omega$-index (cf. [20]). By the assumption $\operatorname{det}(\gamma(t)-\omega I) \neq 0, \forall t \in\left[t_{0}, t_{1}\right],\left.\phi\right|_{\left[0, t_{1}\right]}$ and $\left.\gamma\right|_{\left[0, t_{1}\right]}$ are homotopic(see Definition 5.3). Therefore by Theorem 5.6 (Theorem 2.14 of [20]), (3.6) holds.

Proof of the Proposition 3.5. - Let $F_{\lambda}(x)=(1-\lambda) H(x)+\lambda G(x)$, then $F_{\lambda} \in \mathcal{H}(\Sigma) .\left(\tau_{\lambda}, x_{\lambda}\right)$ is defined as in Lemma 3.2. $\tau_{0}=\tau, \tau_{1}=\mu, x_{0}=$ $x, x_{1}=z_{\sigma}$. Let $R_{F_{\lambda}}(t)$ be the fundamental solution of

$$
\dot{y}=J B_{\lambda}(t) y
$$

where $B_{\lambda}(t)=F_{\lambda}^{\prime \prime}\left(x_{\lambda}(t)\right)$. By the definition of $\mathcal{H}(\Sigma)$, we can suppose

$$
H^{\prime}(x)=\eta(x) G^{\prime}(x), \quad \forall x \in \Sigma,
$$

Vol. 16, $n^{\circ}$ 6-1999. 
where $\eta(x)$ is a positive $C^{1}$-function of $x$ defined on $\Sigma$. Then there hold

$$
H^{\prime}(x)=\eta_{\lambda}(x) F_{\lambda}^{\prime}(x) \text { with } \eta_{\lambda}(x)=\frac{\eta(x)}{(1-\lambda) \eta(x)+\lambda} .
$$

By direct computation, we obtain

$$
\begin{gathered}
x_{\lambda}(l)=x\left(\sigma_{\lambda}^{-1}(l)\right) \text { with } \sigma_{\lambda}(s)=\int_{0}^{s} \eta_{\lambda}(x(t)) d t \\
\tau_{\lambda}=\int_{0}^{\tau} \eta_{\lambda}(x(t)) d t .
\end{gathered}
$$

where $(\tau, x)$ is a $\tau$-periodic solution of the system (3.1) with the Hamiltonian function $H=F_{0}$. Since $F(\lambda, x):=F_{\lambda}(x)$ is $C^{2}$ depending on $x$ and $C^{\infty}$ depending on $\lambda$, so $x_{\lambda}(t)$ and $B_{\lambda}(t)$ are continuous in $\lambda$. Thus $\tau_{\lambda}$ and $\gamma_{\lambda}:=R_{F_{\lambda}}$ are continuous in $\lambda$. By the condition $\omega \notin \sigma\left(R_{I I}(\tau)\right)$, Lemmas 3.2, 3.3 and the above discussion, there holds $\omega \notin \sigma\left(\gamma_{\lambda}\left(\tau_{\lambda}\right)\right)$ for all $\lambda \in[0,1]$. Define $\Gamma(\lambda, s)=\gamma_{\lambda}\left(\tau_{\lambda} s\right)$. Then $\Gamma:[0,1] \times[0,1] \rightarrow \operatorname{Sp}(2 n)$ is an $\omega$-homotopy in the sense of [20] (see Definition 5.3). Thus by Theorem 5.6, $\Gamma(0, \cdot)$ and $\Gamma(1, \cdot)$ have the same $\omega$-index. Since $\Gamma(0, \cdot)$ and $\Gamma(1, \cdot)$ are rescalings of $\gamma_{0}(\cdot)$ and $\gamma_{1}(\cdot)$ respectively, so $\gamma_{0}$ and $\gamma_{1}$ have the same $\omega$-index. Therefore (3.5) holds.

Proof of the Theorem 3.1. - From Proposition 5.6 and Proposition 3.5, there holds

$$
\begin{aligned}
\hat{i}(\tau, x, H) & =\frac{1}{2 \pi} \int_{0}^{2 \pi} i_{\tau,+\sqrt{-1} \theta}(H) d \theta \\
& =\frac{1}{2 \pi} \int_{0}^{2 \pi} i_{\mu, e \sqrt{-1 \theta}}(G) d \theta=\hat{i}\left(\mu, z_{\sigma}, G\right) .
\end{aligned}
$$

If $\Sigma \in \mathcal{E}\left(\mathbb{R}^{2 n}\right)$ is a strictly convex hypersurface of $\mathbb{R}^{2 n}$, and $H \in \mathcal{H}(\Sigma)$ with $H^{\prime \prime}(x)$ positive definite for all $x \in \Sigma$. To understand the relation between the Maslov-type index and the Ekeland index (cf. [10]), note that in [10] the standard symplectic matrix has a sign difference from ours defined in (1.2). So we need to consider the following Hamiltonian system

$$
\dot{x}=-J H^{\prime}(x) \text {. }
$$

If $(\tau, x)$ is a $\tau$-periodic solution of $(3.1)$, then $(\tau, \bar{x})$ with $\bar{x}(t)=x(-t)$ is a $\tau$-periodic solution of (3.8). The linearized system of (3.8) at the periodic solution $\bar{x}$ is

$$
\dot{y}=-J H^{\prime \prime}(x(-t)) y .
$$


In general, we consider the following linear Hamiltonian system

$$
\dot{y}=J B(t) y, \quad y \in \mathbb{R}^{2 n},
$$

where $B(t)$ is a real $2 n \times 2 n \tau$-periodic continuous symmetric matrix. Let $\gamma_{B}(t)$ be the fundamental solution of (3.10), it is well known that $\gamma_{B}(t)$ is a symplectic path starting from identity matrix. Let $\bar{B}(t)=-B(-t)$, and $\gamma_{\bar{B}}(t)$ be the fundamental solution of the system (3.10) with the coefficient $\bar{B}(t)$. Denote by $\left(i_{\tau}(B), \nu_{\tau}(B)\right)$ and $\left(i_{\tau}(\bar{B}), \nu_{\tau}(\bar{B})\right)$ the corresponding Maslov-type indices for $\gamma_{B}$ and $\gamma_{\bar{B}}$ respectively.

Proposition 3.7. - There holds

$$
\begin{gathered}
i_{\tau}(B)+i_{\tau}(\bar{B})+\nu_{\tau}(B)=0, \\
\nu_{\tau}(B)=\nu_{\tau}(\bar{B}) .
\end{gathered}
$$

Proof. - Since $\gamma_{B}(t+\tau)=\gamma_{B}(t) \gamma_{B}(\tau)$, there holds $I=\gamma_{B}(0)=$ $\gamma_{B}(-\tau) \gamma_{B}(\tau)$, then we have $\gamma_{B}(-\tau)=\gamma_{B}(\tau)^{-1}$. From this we have

$$
\nu_{\tau}(B)=\operatorname{dim} \operatorname{ker}\left(\gamma_{B}(\tau)-I\right)=\operatorname{dim} \operatorname{ker}\left(\gamma_{B}(\tau)^{-1}-I\right)=\nu_{\tau}(\bar{B}) .
$$

So (3.12) holds. To prove (3.11) we take the the Hilbert space $E=$ $W^{\frac{1}{2}, 2}\left(S_{\tau}, \mathbb{R}^{2 n}\right)$ with norm $\|\cdot\|$ and inner product $\langle\cdot \cdot \cdot\rangle$, and define operators $A, B, \quad$ in $E$ by

$$
\begin{aligned}
\langle A x, x\rangle & =\int_{0}^{\tau}(-J \dot{x}, x) d t, \\
\langle B x, x\rangle & =\int_{0}^{\tau}(B(t) x, x) d t,
\end{aligned}
$$

and $\bar{B}$ similarly defined as $B$ corresponding to $\bar{B}(t)$. For all $x(t) \in E$ then $\bar{x}(t)=x(-t) \in E$, there hold

$$
\begin{aligned}
\langle A \bar{x}, \bar{x}\rangle & =\int_{0}^{\tau}(J \dot{x}(-t), x(-t)) d t \\
& =\int_{0}^{\tau}(J \dot{x}(t), x(t)) d t \\
& =-\langle A x, x\rangle,
\end{aligned}
$$

and

$$
\begin{aligned}
\langle\bar{B} \bar{x}, \bar{x}\rangle & =\int_{0}^{\tau}(\bar{B}(t) x(-t), x(-t)) d t \\
& =\int_{0}^{-\tau}(B(t) x(t), x(t)) d t \\
& =-\langle B x, x\rangle
\end{aligned}
$$

Vol. $16, n^{\circ} 6-1999$. 
So by definition, the spectral set satisfies

$$
\sigma(A-\bar{B})=-\sigma(A-B)
$$

By Lemma 2.1, for $d>0$ and large number $m$ we have

$$
\begin{gathered}
\operatorname{dim} M_{d}^{+}\left(P_{m}(A-B) P_{m}\right)=\frac{1}{2} \operatorname{dim}\left(P_{m} E\right)-i_{\tau}(B)-\nu_{\tau}(B), \\
\operatorname{dim} M_{d}^{-}\left(P_{m}(A-\bar{B}) P_{m}\right)=\frac{1}{2} \operatorname{dim}\left(P_{m} E\right)+i_{\tau}(\bar{B}),
\end{gathered}
$$

where $B$ and $\bar{B}$ is the operators corresponding to $B(t)$ and $\bar{B}(t)$ defined by (3.14) respectively, and $P_{m}, M_{d}^{ \pm}(\cdot)$ are defined as in Lemma 2.1 . Now (3.17), (3.18) and (3.19) yield (3.11).

CoRollary 3.8. - Suppose $(\tau, x)$ is a $\tau$-periodic solution of (3.1), then $(\tau, \bar{x})$ with $\bar{x}(t)=x(-t)$ is a $\tau$-periodic solution of (3.8) and there hold

$$
\begin{gathered}
i_{\tau}(x)+i_{\tau}(\bar{x})+\nu_{\tau}(x)=0, \\
\nu_{\tau}(x)=\nu_{\tau}(\bar{x}) .
\end{gathered}
$$

We have the following result

THEOREM 3.9. - If $\Sigma \in \mathcal{S}\left(\mathbb{R}^{2 n}\right)$ is a strictly convex hypersurface in $\mathbb{R}^{2 n}$, and $H \in \mathcal{H}(\Sigma)$ with $H^{\prime \prime}(x)$ positive definite for all $x \in \Sigma$. Let $(\tau, x)$ be the $\tau$-periodic solution of (3.1). Then we have

$$
i_{\tau}^{E}(\bar{x})+n=i_{\tau}(x)
$$

where $\bar{x}(t)=x(-t)$ and $i_{\tau}^{E}(\bar{x})$ is the Ekeland index of $\bar{x}$ defined in [10].

Proof. - By the Theorem 7.3 of [20], there holds

$$
i_{r}(\bar{x})+\nu_{\tau}(\bar{x})=-i_{\tau}^{E}(\bar{x})-n .
$$

This can also be obtained from [3] and (3.11). So (3.22) follows from (3.20), (3.21) and (3.23).

CoRollary 3,10. - If $\Sigma \in \mathcal{E}\left(\mathbb{R}^{2 n}\right)$ is a strictly convex hypersurface, and $H \in \mathcal{H}(\Sigma)$ with $H^{\prime \prime}(x)$ positive definite for all $x \in \Sigma$. Let $(\tau, x)$ be a $\tau$-periodic solution of (3.1). Then there holds

$$
\hat{i}^{E}(\tau, \bar{x}, H)=\hat{i}(\tau, x, H) .
$$

where $\hat{i}^{E}(\tau, \bar{x}, H)$ is the Ekeland mean index per period $\tau$ of $\bar{x}$, and $\hat{i}(\tau, x, H)$ is the Maslov-type mean index per period $\tau$ of $x$.

Proof. - This follows directly from the definitions of Maslov-type mean index, Ekeland mean index, and (3.22). 


\section{PROPERTIES OF HYPERBOLIC CHARACTERISTICS}

For $x \in E$, we define

$$
f(x)=\frac{1}{2} \int_{0}^{1}(-J \dot{x}, x) d t-\int_{0}^{1} H_{4}(x(t)) d t .
$$

It is clear that $f \in C^{2}(E, \mathbb{R})$ and the critical points of $f$ coincide with the solutions of the following problem

$$
\left\{\begin{array}{l}
\dot{x}(t)=J H_{4}^{\prime}(x(t)) \\
x(1)=x(0)
\end{array}\right.
$$

If $x \in E$ is a nontrivial critical point of $f$ defined in (4.1), then $x$ is a nonconstant solution of problem (4.2). Its period is $\tau=1$. We denote the corresponding Maslov-type index of $x$ by $\left(i_{1}(x), \nu_{1}(x)\right)$. Let $h=H_{4}(x(t))$, and define

$$
z(t)=h^{-\frac{1}{4}} x\left(h^{-\frac{1}{2}} t\right)
$$

Then $z(t) \in \Sigma$ for all $t \in \mathbb{R}$ and $z$ is an $h^{\frac{1}{2}}$-periodic solution of the fixed energy problem (2.1) with $\tau=h^{\frac{1}{2}}$.

LEMMA 4.1. - For $z(t), x(t)$ defined above and $\tau=h^{\frac{1}{2}}$, there hold

$$
i_{\tau}(z)=i_{1}(x) \text { and } \nu_{\tau}(z)=\nu_{1}(x)
$$

Proof. - We follow the idea of [21]. Let $\psi:[0,+\infty) \rightarrow \operatorname{Sp}(2 n)$ be the associated symplectic path of $x$, i.e., the fundamental solution of $\dot{y}=J H_{4}^{\prime \prime}(x(t)) y$ with $\psi(0)=I_{2 n}$. We define

$$
\gamma(t)=\psi\left(h^{-\frac{1}{2}} t\right), \quad \forall t \in[0,+\infty)
$$

Then using the positive homogeneity of $H_{4}^{\prime \prime}$ (Its degree is 2), we obtain that $\gamma:[0,+\infty) \rightarrow \operatorname{Sp}(2 n)$ is the fundamental solution of the system

$$
\dot{y}=J H_{4}^{\prime \prime}(z(t)) y \text { with } \gamma(0)=I_{2 n} .
$$

Thus by (3.23) there holds $\gamma(\tau)=\psi(1)$. This implies $\nu_{\tau}(z)=\nu_{1}(x)$. Since $\left.\gamma\right|_{[0, \tau]}$ is only a rescaling of $\left.\psi\right|_{[0,1]}$, they are geometrically the same path in $\operatorname{Sp}(2 n)$. This yields $i_{\tau}(z)=i_{\tau}\left(\left.\gamma\right|_{[0, \tau]}\right)=i_{1}(\psi \mid[0,1])=i_{1}(x)$ and completes the proof. 
REMARK 4.2. - We note that replacing the function $H_{4}$ by function $H_{\alpha}$ with some $\alpha>1$, Lemma 4.1 is still true with $z(t)=h^{-1 / \alpha} x\left(h^{2-c / \alpha} t\right)$ and $\tau=h^{\alpha-2 / \alpha}$.

We consider a nonconstant $\tau$-periodic solution $x$ of the given energy problem:

$$
\left\{\begin{array}{l}
\dot{x}(t)=J H_{4}^{\prime}(x(t)), \quad H_{4}(x(t))=1, \quad \forall t \in \mathbb{R}, \\
x(\tau)=x(0),
\end{array}\right.
$$

and denote by $\gamma_{x}(t)$ the fundamental solution of the linearized system of (4.6) at $x(t)$

$$
\left\{\begin{array}{l}
\dot{y}(t)=J H_{4}^{\prime \prime}(x(t)) y(t), \quad \forall t \in \mathbb{R}, \\
y(\tau)=y(0) .
\end{array}\right.
$$

LEMMA 4.3. - If $x$ is a nonzero $\tau$-periodic solution of (4.6) and $\gamma_{x}$ is the fundamental solution of (4.7), there hold

$$
\begin{gathered}
\gamma_{x}(\tau) \dot{x}(0)=\dot{x}(0), \\
\gamma_{x}(\tau) x(0)=2 \tau \dot{x}(0)+x(0) .
\end{gathered}
$$

Proof. - The proof is similar to that of Lemma I.7.3 in [10], and is omitted.

For

$$
M_{1}=\left(\begin{array}{ll}
A_{1} & B_{1} \\
C_{1} & D_{1}
\end{array}\right)_{2 i \times 2 i}, M_{2}=\left(\begin{array}{ll}
A_{2} & B_{2} \\
C_{2} & D_{2}
\end{array}\right)_{2 j \times 2 j}
$$

being two even order matrices of square block form, we defined the $\diamond$ product of $M_{1}$ and $M_{2}$ to be the $2(i+j) \times 2(i+j)$ matrix $M_{1} \diamond M_{2}$ (cf. [19], [21] [22] or [8])

$$
M_{1} \diamond M_{2}=\left(\begin{array}{cccc}
A_{1} & 0 & B_{1} & 0 \\
0 & A_{2} & 0 & B_{2} \\
C_{1} & 0 & D_{1} & 0 \\
0 & C_{2} & 0 & D_{2}
\end{array}\right)
$$

and $M_{1}^{\diamond k}$ to be the $k$-times $\diamond$-product of $M_{1}$. Note that the $\diamond$-product is associative and the $\diamond$-product of two symplectic matrices is still symplectic.

Lemma 4.4. - For every solution $(x, \tau) \in \mathcal{J}_{4}(\Sigma)$, there exist matrices $P \in \mathrm{Sp}(2 n)$ and $M \in \mathrm{Sp}(2 n-2)$ such that there holds

$$
\gamma_{x}(\tau)=P\left(N_{2}(-1) \diamond M\right) P^{-1}
$$


where we define

$$
N_{2}(a)=\left(\begin{array}{ll}
1 & a \\
0 & 1
\end{array}\right) \in S p(2)^{0}, \quad \forall a \in \mathbb{R} .
$$

Proof. - This lemma was essentially proved in [21]. For reader's convenience we enclose the proof here. Fix $(x, \tau) \in \mathcal{J}_{4}(\Sigma)$, by Lemma 4.3, we have (4.8) and (4.9) Define

$$
\xi_{1}=2 \tau \dot{x}(0), \quad \xi_{2}=x(0)
$$

We carry out the proof in three steps.

Step 1. - Since $x=x(t)$ is a solution of (1.4), we have $x(0) \in \Sigma$ and $\dot{x}(0)=J H_{4}^{\prime}(x(0))$, we obtain

$$
\begin{aligned}
\xi_{1}^{*} J \xi_{2} & =2 \tau \dot{x}(0)^{T} J x(0)=2 \tau H_{4}^{\prime}(x(0))^{T} J^{T} J x(0) \\
& =2 \tau\left(H_{4}^{\prime}(x(0)), x(0)\right)=8 \tau H_{4}(x(0))=8 \tau>0 .
\end{aligned}
$$

Step 2. - Now suppose $\left\{\xi_{1}, \xi_{2}, \cdots, \xi_{p}\right\}$ form a Jordan block of $\gamma_{x}(\tau)$ belonging to eigenvalue 1 , i.e, setting $\xi_{0}=0$, there holds

$$
\gamma_{x}(\tau) \xi_{i}=\xi_{i}+\xi_{i-1}, \quad \forall 1 \leq i \leq p
$$

As in the section 11 of [21] for $1 \leq i, j \leq p$ by (5.2), we have

$$
\xi_{i}^{*} J \xi_{j}=\left(\gamma_{x}(\tau) \xi_{i}\right)^{*} J\left(\gamma_{x}(\tau) \xi_{j}\right)=\xi_{i}^{*} J \xi_{j}+\xi_{i-1}^{*} J \xi_{j}+\xi_{i}^{*} J \xi_{j-1}+\xi_{i-1}^{*} J \xi_{j-1}
$$

This yield

$$
\begin{gathered}
\xi_{i-1}^{*} J \xi_{j-1}+\xi_{i-1}^{*} J \xi_{j}+\xi_{i}^{*} J \xi_{j-1}=0, \quad \forall 1 \leq i, j \leq p, \\
\xi_{i}^{*} J \xi_{j}=0 \quad \forall 1 \leq i \leq p-j, \quad 1 \leq j \leq\left[\frac{p}{2}\right],
\end{gathered}
$$

where $[a]$ is the integer part of $a$ defined by $[a]=\max \{m \in \mathbb{Z} \mid m \leq a\}$ for $a \in \mathbb{R}$, and (4.18) follows from (4.17) by induction.

Thus from (4.8), (4.9) and (4.18) we must have $p=2$, i.e., $\xi_{1}$ and $\xi_{2}$ form a Jordan block of $\gamma_{x}(\tau)$ belonging to the eigenvalue 1 .

Step 3. - Define

$$
\delta_{1}=\frac{1}{\sqrt{8 \tau}} \xi_{1}, \quad \delta_{2}=-\frac{1}{\sqrt{8 \tau}} \xi_{2}
$$

Vol. $16, \mathrm{n}^{\circ}$ 6-1999. 
Then there hold

$$
\delta_{1}^{*} J \delta_{2}=-1, \quad F \equiv \operatorname{span}\left\{\delta_{1}, \delta_{2}\right\}=\operatorname{span}\left\{\xi_{1}, \xi_{2}\right\}
$$

i.e., $\left\{\delta_{1}, \delta_{2}\right\}$ form a symplectic base for $F$. Denote by $K$ the $2 n \times 2$ matrix formed by $\delta_{1}$ and $\delta_{2}$ as the first and the second columns. From (4.14), (4.15), (4.19) and (4.20), we obtain $\gamma_{x}(\tau) K=K N_{2}(-1)$. Now we can extend $K$ to a matrix $P \in \mathrm{Sp}(2 n)$ such that $\delta_{1}$ and $\delta_{2}$ form the first and the $(n+1)$-st columns of $P$ and for some $M \in \mathrm{Sp}(2 n-2)$ such that (4.11) holds.

Lemma 4.5. - Suppose $(x, \tau) \in \mathcal{J}_{4}(\Sigma)$ is hyperbolic. Then

$$
i_{m \tau}\left(x^{m}\right)=m i_{\tau}(x), \quad \nu_{m \tau}\left(x^{m}\right)=1, \quad \forall m \in \mathbb{N}
$$

Proof. - The proof follows from the argument in [21] and the above Lemma 4.4 .

LEMMA 4.6. - Suppose there are only finitely many closed characteristics on $\Sigma$, and all of them are hyperbolic with their mean Maslov-type index greater than 0 . Then for every $k \in \mathbb{N}$, there exists a solution $\left(x_{k}, \tau_{k}\right)$ of (4.6) for some $\tau_{k}>0$ with its Maslov-type index satisfying

$$
i_{\tau_{k}}\left(x_{k}\right)=n+2 k-1
$$

Proof. - The essential ideas of this proof come from [27]. We use the notations defined in [27].

As in Lemma 2.2 of [27], we choose a function $\phi: \mathbb{R}_{+} \rightarrow \mathbb{R}$ such that $\phi$ is $C^{3}$, nonnegative, $\phi(t)=\frac{a}{4} t^{2}$ near the origin. Further more, we need $\phi(t)=t^{\alpha}$ in the interval $(\delta, A)$ for $\delta>0$ small and $A>0$ large enough to be chosen below, where $\alpha=\alpha(a) \in(1,2)$ will be very close to 2 . More precisely, the following equation

$$
\frac{a t^{2}}{4}=t^{\alpha}
$$

has a solution $t_{0}=(4 / a)^{1 /(2-\alpha)}$. For large $a$, we can choose it suitably so that there is an open neighborhood $U\left(\frac{a T}{2}, r\right)$ of $\frac{a T}{2}$ with radius $r>0$ such that $j \tau \notin U\left(\frac{a T}{2}, r\right)$ for all $j \in \mathbb{N}$ and every $\tau$ which is the minimal period of some periodic solution on $\Sigma$. Here $T$ is fixed and defined in (3.3) of [27]. Then we choose $\alpha<2$ and close to 2 such that $\frac{a}{2}-\frac{a \alpha}{4}=\frac{a}{4}(2-\alpha)<\frac{r}{2}$. 
Now we define $\phi(t)$. When $0<t \leq t_{0} / 2$, we define $\phi(t)=\frac{a t^{2}}{4}$, so $g(t):=\phi^{\prime}(t) / t=\frac{u}{2}$. When $t \geq t_{0}$, we define $\phi(t)=t^{\alpha}$, so $g(t)=\phi^{\prime}(t) / t=\alpha t^{\alpha-2}$ and $g\left(t_{0}\right)=\phi^{\prime}\left(t_{0}\right) / t_{0}=\frac{a \alpha}{4}$. Since $g\left(t_{0} / 2\right)$ and $g\left(t_{0}\right)$ are contained in $U\left(\frac{a}{2}, \frac{r}{2}\right)$, we can connect $\phi(t)$ from $t_{0} / 2$ to $t_{0}$ such that $\phi^{\prime}(t) / t \in U(a / 2, r)$ for $0<t \leq t_{0}$. From this definition, we note that $\phi^{\prime}(t) / t$ is decreasing when $t>t_{0}$ and $\phi^{\prime}(t) / t \rightarrow 0$ as $t \rightarrow+\infty$. Then we define $\delta=t_{0}$.

Set $\tilde{H}(x)=\phi\left(j_{\Sigma}(x)\right)$, and $U_{A}=\{x \mid \tilde{H}(x) \leq A\}$ for some large $A$. Following [27], we now truncate the function $\tilde{H}(x)$ near the infinity by constructing a function $H$, coinciding with $\tilde{H}$ on $U_{A}$, with $\frac{1}{2} \varepsilon|x|^{2}$ outside some large ball, such that $H^{\prime}(x)$ does not vanish and $\left|H^{\prime \prime}(x)\right|<\varepsilon$ outside $U_{A}$ (see p. 624 of [27] for details).

We now consider the following Hamiltonian system with the $T$ fixed above,

$$
\left\{\begin{array}{l}
\dot{x}(t)=J H^{\prime}(x(t)), \\
x(0)=x(T) .
\end{array}\right.
$$

Since $j \tau \notin U(a T / 2, r)$ for all $j \in \mathbb{N}$ and $\tau$ being the minimal period of any solution on $\Sigma$, and $\phi^{\prime}(t) / t \in U(a / 2, r)$ for $0<t \leq t_{0}$, by Lemmas 2.1 and 2.2 of [27] there is no $T$-periodic solution of (4.24) in the domain $\left\{x \in \mathbb{R}^{2 n} \mid 0<H(x) \leq \delta\right\}$. Now we choose $\varepsilon>0$ small enough so that $\varepsilon T<2 \pi$. Then by Yorke's Theorem (cf. [30]), there is no $T$-periodic solution of (4.24) outside the domain $U_{A}$. Since (4.24) is autonomous, all nontrivial solutions of (4.24) must be contained in the domain $\left\{x \in \mathbb{R}^{2 n} \mid \delta<H(x) \leq A\right\}$. It means that it is a solution of the following Hamiltonian system

$$
\left\{\begin{array}{l}
\dot{x}(t)=J H_{\alpha}^{\prime}(x(t)) \\
x(0)=x(T)
\end{array}\right.
$$

where $H_{\alpha}=j_{\Sigma}(x)^{\alpha}$ with $\alpha \in(1,2)$ and close to 2 .

Since all the periodic solutions on $\Sigma$ are hyperbolic, all the solutions of (4.25) are also hyperbolic. By Proposition 4.1 of [21] and $1<\alpha<2$, the nullity of such a solution must be 1 . Therefore, all solution orbits of (4.25) are non-degenerate critical manifolds in the sense of R. Bott.

Since there are only finitely many closed characteristics on $\Sigma$, and by the condition $\hat{i}(x)>0$ for all solution $x$ on $\Sigma$, from [27], for a large enough, there holds (see (8.12) of [27])

$$
M(t)-\frac{1}{1-t^{2}}=(1+t) U(t)
$$

Vol. $16, n^{\circ}$ 6-1999. 
where $M(t)$ is the equivariant Morse series for nondegenerate critical orbits of the functional $F_{K}$ (defined in (3.3) of [27]) in $X^{-\eta}-X^{-4}$ for some small $\eta>0$ and large $b>0, X^{s}=\left\{x \in E \mid F_{K}(x) \leq s\right\}$, and $U(t)$ is a series with nonnegative coefficients. From (4.26) and our above discussions, for each $k \in \mathbb{N}$, we obtain a nondegenerate critical orbit $u_{k}$ of $F_{K}$ with Morse index $d(K)+2(k-1)$, where $d(K)$ is defined by lemma 5.2 of [27]. The index defined by Lemma 6.4 of [27] of $u_{k}$. is

$$
i_{T}^{\prime \prime}\left(u_{k}\right)=2 k-2, \quad \nu_{T}^{*}\left(u_{k}\right)=1
$$

By the same reason of Lemma 1.3 of [21] and [3], we have the Maslov-type index of $u_{k}$ satisfies

$$
i_{T}\left(u_{k}\right)=i_{T}^{v}\left(u_{k}\right)+n=2 k-2+n . \quad \nu_{T}\left(u_{k}\right)=\nu_{T}^{v}\left(u_{k}\right)=1 .
$$

By the rescaling given in Remark 4.2, from this $u_{k}$, we obtain a solution $\left(z_{k}, \mu_{k}\right)$ on $\Sigma$. By Lemma $4.1, z_{k}$ possesses the same Maslov-type index with that of $u_{k}$, i.e.,

$$
i_{\mu_{k}}\left(z_{k}\right)=i_{T}\left(u_{k}\right)=2 k-2+n, \quad \nu_{\mu_{k}}\left(z_{k}\right)=\nu_{T}\left(u_{k}\right)=1 .
$$

By further rescaling from $z_{k}$ as in [15] (or an analogue of Proposition I.7.5 of [10]), we get a solution $\left(x_{k}, \tau_{k}\right)$ of (4.6) with Hamiltonian function $H_{4}(x)$ such that

$$
i_{\tau_{k}}\left(x_{k}\right)=i_{\mu_{k}}\left(z_{k}\right)+1=2 k-1+n, \quad \nu_{\tau_{k}}\left(x_{k}\right)=\nu_{\mu_{k}}\left(z_{k}\right)=1 .
$$

This completes the proof of the lemma.

Now we can give the proof of our main result in this paper.

Proof of Theorem 1.2. - We prove the theorem indirectly by assuming there are only finitely many closed characteristics on $\Sigma$, and all of them are hyperbolic. Let $(x, \tau) \in \mathcal{J}_{4}(\Sigma)$ with minimal period $\tau$, then by (4.21), we have $\hat{i}_{\tau}(x)=i_{\tau}(x)$, and $i_{m \tau}\left(x^{m}\right)=m \hat{i}_{\tau}(x)$. Thus the Maslov-type indices of all periodic solutions of (4.6) must be contained in the set $\left\{m q \mid m \in \mathbb{N}, q_{n}<q \leq q_{0}\right\}$ for some integer $q_{0}$, where $q_{n}=\frac{3-(-1)^{n}}{2}$. By the assumption of the theorem, applying Lemma 4.6, we obtain

$$
Q(n):=\{2 k-1+n \mid k \in \mathbb{N}\} \subset\left\{m q \mid m \in \mathbb{N}, q_{n}<q \leq q_{0}\right\}
$$

We now consider two cases according to the parity of $n$. 
If $n$ is odd, we can choose a prime number $p>\max \left\{q_{0}, n\right\}$, and define $k$ by $2 k-1=2 p-n$, i.e., $2 p=n+2 k-1 \in Q(n)$. Thus there are integers $m$ and $q \in\left(2, q_{0}\right]$ such that $2 p=m q>2 q_{0}$. So we must have $m>2$. But we also have $q>2$, this contradicts to the choice of $p$.

If $n$ is even, we can choose a prime number $p>\max \left\{q_{0}, n\right\}$, and define $k$ by $2 k-1=p-n$, i.e., $p=n+2 k-1 \in Q(n)$. Thus there exist integers $m$ and $q \in\left(1, q_{0}\right]$ such that $p=m q>q_{0}$, so we have $m>1$. This contradicts to the choice of $p$.

\section{APPENDIX. \\ THE $\omega$-INDEX THEORY FOR SYMPLECTIC PATHS}

The $\omega$-index theory for continuous symplectic paths starting from the identity matrix $I$ was first established in [20]. In this section we give a brief introduction of this $\omega$-index theory without proofs. For details we refer to [20]. Denote by

$$
\mathcal{P}_{\tau}(2 n)=\left\{\gamma \in C([0, \tau], \operatorname{Sp}(2 n)) \mid \gamma(0)=I_{2 n}\right\} .
$$

For any $\omega \in \mathbf{U}$, the unite circle in complex plane, and $M \in \mathrm{Sp}(2 n)$, define

$$
D_{\omega}(M)=(-1)^{n-1} \omega^{-n} \operatorname{det}(M-\omega I) .
$$

One can easily see that $D_{\omega}=D_{\bar{\omega}}$ for all $\omega \in \mathbf{U}$ and $D \in C^{\infty}(\mathbf{U} \times$ $\operatorname{Sp}(2 n), \mathbb{R})$.

DEFINITION 5.1. - For $\omega \in \mathbf{U}$ we define

$$
\begin{aligned}
& \mathrm{Sp}(2 n)_{\omega}^{ \pm}=\left\{M \in \mathrm{Sp}(2 n) \mid \pm D_{\omega}(M)<0\right\}, \\
& \mathrm{Sp}(2 n)_{\omega}^{*}=\operatorname{Sp}(2 n)_{\omega}^{+} \cup \mathrm{Sp}(2 n)_{\omega}^{-}, \\
& \mathrm{Sp}(2 n)_{\omega}^{\circ}=\operatorname{Sp}(2 n) \backslash \mathrm{Sp}(2 n)_{\omega}^{*} .
\end{aligned}
$$

Let $H(a)=\operatorname{diag}\left(a, a^{-1}\right)$ for $a \in \mathbb{R} \backslash\{0\}$. Using (4.10) we define

$$
M_{n}^{+}=H(2)^{\diamond n}, M_{n}^{-}=H(-2) \diamond H(2)^{\diamond(n-1)},
$$

and

$$
\mathcal{P}_{\tau, \omega}^{*}(2 n)=\left\{\gamma \in \mathcal{P}_{\tau}(2 n) \mid \gamma(\tau) \in \mathrm{Sp}(2 n)_{\omega}^{*}\right\}
$$

DefinItion 5.2. - For any $\tau>0$ and $\gamma \in \mathcal{P}_{\tau}(2 n)$, we define

$$
\nu_{\tau, \omega}(\gamma)=\operatorname{dim}_{\mathbb{C}} \operatorname{ker}_{\mathbb{C}}(\gamma(\tau)-\omega I), \quad \forall \omega \in \mathbf{U}
$$

Vol. 16, $n^{\circ}$ 6-1999. 
Definition 5.3. - for $\tau>0$ and $\omega \in \mathbf{U}$, given two paths $\gamma_{0}$ and $\gamma_{1} \in \mathcal{P}_{\tau}(2 n)$, if there exists a map $\delta \in C([0,1] \times[0, \tau], \mathrm{Sp}(2 n))$ such that $\delta(0, \cdot)=\gamma_{0}(\cdot), \delta(1, \cdot)=\gamma_{1}(\cdot), \delta(s, 0)=I$ and $\nu_{\tau, \omega}(\delta(s, \cdot))$ is constant for $0 \leq s \leq 1$, then $\gamma_{0}$ and $\gamma_{1}$ are $\omega$-homotopic on $[0, \tau]$ along $\delta(\cdot, \tau)$ and we write $\gamma_{0} \sim_{\omega} \gamma_{1}$. If $\gamma_{0} \sim_{\omega} \gamma_{1}$ for all $\omega \in U$, then $\gamma_{0}$ and $\gamma_{1}$ are homotopic on $[0, \tau]$ along $\delta(\cdot, \tau)$ and we write $\gamma_{0} \sim \gamma_{1}$.

As well known, every $M \in \mathrm{Sp}(2 n)$ has its unique polar decomposition $M=A U$, where $A=\left(M M^{T}\right)^{1 / 2}$, and $U$ has the form

$$
U=\left(\begin{array}{cc}
u_{1} & -u_{2} \\
u_{2} & u_{1}
\end{array}\right)
$$

and $u=u_{1}+\sqrt{-1} u_{2} \in \mathcal{L}\left(\mathbb{C}^{n}\right)$ is a unitary matrix. So there exists a continuous real function $\Delta(t)$ satisfying $\operatorname{det} u(t)=\exp (\sqrt{-1} \Delta(t))$, and define $\Delta_{\tau}(\gamma)=\Delta(\tau)-\Delta(0) \in \mathbb{R}$

For any $\gamma \in \mathcal{P}_{\tau, \omega}^{*}(2 n)$, we can connect $\gamma(\tau)$ to $M_{n}^{-}$or $M_{n}^{+}$by a path $\beta$ within $\operatorname{Sp}(2 n)_{\omega}^{*}$ and get a product path $\beta * \gamma$ defined by $\beta * \gamma(t)=\gamma(2 t)$ if $0 \leq t \leq \tau / 2, \beta * \gamma(t)=\beta(2 t-\tau)$ if $\tau / 2 \leq t \leq \tau$. Then

$$
k \equiv \frac{1}{\pi} \Delta_{\tau}(\beta * \gamma) \in \mathbb{Z}
$$

In this case, we define

$$
i_{\tau, \omega}(\gamma)=k \in \mathbb{Z}
$$

For $\gamma \in \mathcal{P}_{\tau, \omega}^{0}(2 n):=\mathcal{P}_{\tau}(2 n) \backslash \mathcal{P}_{\tau, \omega}^{*}(2 n)$, define

$i_{\tau, \omega}(\gamma)=\inf \left\{i_{\tau, \omega}(\beta) \mid \beta \in \mathcal{P}_{\tau}^{*}(2 n)\right.$ and $\beta$ is $C^{0}$-close enough to $\left.\gamma\right\}$.

THEOREM 5.4. - For any $\gamma \in \mathcal{P}_{\tau}(2 n)$, the above definition yields

$$
\left(i_{\tau, \omega}(\gamma), \nu_{\tau, \omega}(\gamma)\right) \in \mathbb{Z} \times\{0,1, \cdots, 2 n\},
$$

which are called the $\omega$-index of $\gamma$.

For any $\gamma \in \mathcal{P}_{\tau}(2 n)$, define the iteration path $\tilde{\gamma} \in C([0,+\infty), \mathbf{S p}(2 n))$ of $\gamma$ by

$$
\tilde{\gamma}(t)=\gamma(t-j \tau) \gamma(\tau)^{j}, \quad \text { for } j \tau \leq t \leq(j+1) \tau \text { and } j \in\{0\} \cup \mathbb{N} \text {. }
$$

THEOREM 5.5. - For any $\gamma \in \mathcal{P}_{\tau}(2 n)$ and $k \in \mathbb{N}$,

$$
i_{k \tau}(\tilde{\gamma})=\sum_{\omega^{k}=1} i_{\tau, \omega}(\gamma), \quad \nu_{k \tau}(\tilde{\gamma})=\sum_{\omega^{k}=1} \nu_{\tau, \omega}(\gamma)
$$




$$
\hat{i}_{\tau}(\gamma):=\lim _{k \rightarrow \infty} \frac{i_{k \tau}(\tilde{\gamma})}{k}=\frac{1}{2 \pi} \int_{0}^{2 \pi} i_{\tau, \exp (\sqrt{-1} \theta)}(\gamma) d \theta \in \mathbb{R}
$$

which is called the mean index per period $\tau$ of $\gamma \in \mathcal{P}_{\tau}(2 n)$

THEOREM 5.6 (Homotopy invariant). - For any two paths $\gamma_{0}$ and $\gamma_{1} \in \mathcal{P}_{\tau}(2 n)$, if $\gamma_{0} \sim_{\omega} \gamma_{1}$ on $[0, \tau]$, there hold

$$
i_{\tau, \omega}\left(\gamma_{0}\right)=i_{\tau, \omega}\left(\gamma_{1}\right), \quad \nu_{\tau, \omega}\left(\gamma_{0}\right)=\nu_{\tau, \omega}\left(\gamma_{1}\right)
$$

THEOREM 5.7 (Symplectical additivity). $-\forall \gamma_{j} \in \mathcal{P}_{\tau}\left(2 n_{j}\right), n_{j} \in \mathbb{N}, \quad j=$ 0 , 1, there holds

$$
i_{\tau, \omega}\left(\gamma_{0} \diamond \gamma_{1}\right)=i_{\tau, \omega}\left(\gamma_{0}\right)+i_{\tau, \omega}\left(\gamma_{1}\right)
$$

\section{REFERENCES}

[1] A. BAHRI and H. BERESTYCKI, Forced vibrations of superquadratic Hamiltonian systems, Acta Mathematica, Vol. 152, 1984, pp. 143-197.

[2] H. Berestycki, J. M. LasRy, G. Mancini and B. Rof, Existence of multiple periodic orbits on starshaped Hamiltonian systems, Comm. pure Appl. Math., Vol. 38, 1985 , pp. 253-289.

[3] V. Brousseau, Espaces de Krein et index des systémes hamiltoniens, Ann. Inst. H. Poincaré, Anal. non linéaire, Vol. 7, 1990, pp. 525-560.

[4] K. C. CHANG, Infinite dimensional Morse theory and multiple solution problems, Birkhäuser, Boston, 1993.

[5] C. CONLEY and E. ZEHNDER, Maslov-type index theory for flows and periodic solutions for Hamiltonian equations, Commun. Pure Appl. Math., Vol. 37, 1984, pp. 207-253.

[6] G. Dell'Antonio, Variational calculus and stability of periodic solutions of a class of Hamiltonian systems, SISSA Ref. (185/92/FM (Oct. 1992)).

[7] G. Dell' Antonio, B. D'Onofrio and I. EkEland, Les systëm hamiltoniens convexes et pairs ne sont pas ergodiques en general, C. R. Acad. Sci. Paris, t. 315, Series I, 1992, pp. 1413-1415.

[8] D. Dong and Y. Long, The Iteration Formula of the Maslov-type Index Theory with Applications to Nonlinear Hamiltonian Systems, Trans. Amer. Math. Soc., Vol. 349, 1997, pp. 2619-2661.

[9] I. EKELAND and H. Hofer, Convex Hamiltonian energy surfaces and their closed trajectories, Comm. Math. Physics, Vol. 113, 1987, pp. 419-467.

[10] I. Ekeland, Convexity Methods in Hamiltonian Mechanics, Springer, Berlin, 1990.

[11] I. Ekeland, An index theory for periodic solutions of convex Hamiltonian systems, In Nonlinear Funct. Anal. and its Appl. Proc. Symposia in pure Math., Vol. 45-1, 1986, pp. 395-423.

[12] I. Ekeland, Une thórie de Morse pour les systèms hamiltoniens convexes, Ann. Inst. Henri poincaté. Anal. Non Linéair, Vol. 1, 1984, pp. 19-78.

[13] G. FEI and Q. QIu, Periodic solutions of asymptotically linear Hamiltonian systems, Preprint, 1996, Chinese Ann. of Math. (To appear).

[14] N. Ghoussous, Location, multiplicity and Morse indices of minimax critical points, J. Reine Angew Math., Vol. 417, 1991, pp. 27-76.

[15] C. Liu, Monotonicity of the Maslov-type index and the w-index theory, Acta of Nankai University (Chinese). to appear. 
[16] C. LIU and Y. Long, An optimal increasing estimate of the Maslov-type indices for iterations, Chinese Sci. Bull, Vol. 42, 1997, pp. 2275-2277 (Chinese edition), Vol. 43, 1998, pp. $1063-1066$ (English edition).

[17] Y. LoNG, Maslov-type index, degenerate critical points, and asymptotically linear Hamiltonian systems, Science in China (Scientia Sinica), Vol. Series A.33, 1990, pp. 1409-1419.

[18] Y. LoNG, A Maslov-type theory and asymptotically linear Hamiltonian systems, In Dvna. Syst. and Rel. Topics. K. Shiraaiwa ed. World Sci., 1991, pp. 333-341.

[19] Y. LONG, The Index Theory of Hamiltonian Systems with Applications, (In Chinese) Science Press, Beijing, 1993.

[20] Y. LONG, Bott formula of the Maslov-type index theory, Nankai Inst. of Math. Nankai Univ. Preprint, 1995, Revised 1996, 1997, Pacific.J. Math., Vol. 187, 1999, pp. 11.3-149.

[21] Y. LONG, Hyperbolic closed characteristics on compact convex smooth hypersurfaces in $\mathbb{R}^{2 n}$, Nankai Inst. of Math. Preprint, 1996, Revised 1997, J. Diff. Equa., Vol. 150, 1998. pp. $227-249$.

[22] Y. Long, A Maslov-type index theory for symplectic paths, Top. Meth. Nonl. Anal., Vol. 10, 1997, pp. 47-78.

[23] Y. LONG and E. ZEHNDER, Morse theory for forced oscillations of asymptotically linear Hamiltonian systems, Stoc. Proc. Phys. and Geom., S. Albeverio et al. ed. World Sci., 1990, pp. 528-563.

[24] P. H. Rabinowitz, Periodic solutions of Hamiltonian systems, Comm. Pure Appl. Math., Vol. 31, 1978, pp. 157-184.

[25] P. H. RaBINOWITZ, Minimax methods in critical point theory with applications to differential equations, CBMS Regional Conf. Ser. in Math. Amer. Math. Soc., Vol. 65, 1986.

[26] C. Viterbo, A Proof of the Weinstein conjecture in $\mathbb{R}^{2 n}$, Ann. IHP. Analyse nonlinéaire, Vol. 4, 1987, pp. 337-357.

[27] C. Viterbo, Equivariant Morse theory for starshaped Hamiltonian systems, Trans. Amer. Math. Soc., Vol. 311, 1989, pp. 621-655.

[28] T. WANG and G. FEI, Subharmonics for superquadratic Hamiltonian systems via the iteration method of the Maslov-type index theory, Preprint, 1996.

[29] A. WeinsteIn, On the Hypotheses of Rabinowitz' Periodic Orbit Theorems, J. Diff. Equa., Vol. 33, 1979, pp. 353-358.

[30] J. A. YURke, periods of periodic solutions and the Lipschili contanl, Proc. Amer. Math. Soc., Vol. 22, 1969, pp. 509-512.

(Manuscript received November 4, 1998.) 